\title{
Comparative metabolomics analysis of donkey colostrum and mature milk using ultra-high-performance liquid tandem chromatography quadrupole time-of-flight mass spectrometry
}

\author{
Mohan Li, ${ }^{1} \odot$ Shimo Kang, ${ }^{1}$ Yan Zheng, ${ }^{1}$ Junhua Shao, ${ }^{1}$ Huiwen Zhao, ${ }^{1}$ Yuejia An, ${ }^{1}$ Ge Cao, ${ }^{1}$ Qilong Li, ${ }^{2}$ \\ Xiqing Yue, ${ }^{1 *}$ () and Mei Yang ${ }^{1 *}$ \\ ${ }^{1}$ College of Food Science, Shenyang Agricultural University, Shenyang, Liaoning Province, China 110866 \\ ${ }^{2}$ College of Bioscience and Biotechnology, Shenyang Agricultural University, Shenyang, Liaoning Province, China 110866
}

\begin{abstract}
Donkey milk has been widely shown to be an ideal substitute for human milk because of its similar composition. However, alterations to the composition of donkey milk during lactation have not been well studied. In this study, untargeted metabolomics with ultra-high-performance liquid tandem chromatography quadrupole time-of-flight mass spectrometry were used to analyze and compare the metabolites in donkey colostrum (DC) and mature milk (DMM). Two hundred seventy metabolites were characterized in both DC and DMM. Fifty-two of the metabolites in the DC were significantly different from those in the DMM; 8 were downregulated and 44 were upregulated. This demonstrated that the composition of the donkey milk changed with lactation. Additionally, the interactions and metabolic pathways were further analyzed to explore the mechanisms that altered the milk during lactation. Our results provide comprehensive insights into the alterations in donkey milk during lactation. The results will aid in future investigations into the nutrition of donkey milk and provide practical information for the dairy industry.
\end{abstract}

Key words: colostrum, donkey milk, lactation, metabolomics

\section{INTRODUCTION}

Human milk is generally an infant's first and exclusive food, and is promoted as the gold standard in neonatal nutrition (Scano et al., 2016; Cao et al., 2018).

Received August 13, 2019.

Accepted September 13, 2019.

*Corresponding authors: yxqsyau@126.com and 18624061478@163 .com
Selecting an alternative milk supply, especially when breastfeeding is not allowed, is thus important for the health of the baby. Donkey milk, because of its similarity to human milk, has been widely suggested to be an ideal substitute (Chiofalo et al., 2011; Martemucci and D'Alessandro, 2012). Several studies have shown that donkey milk has certain physiological functions, such as high tolerability (Polidori and Vincenzetti, 2013), antimicrobial activities (Zhang et al., 2008), and anticancer activities (Mao et al., 2009).

The composition of milk has been proven to be affected by a combination of factors including the environment, season, diet, physiological state, lactation period, and so on (Guo et al., 2001; Zhang et al., 2005; Martemucci and D'Alessandro, 2012; Martini et al., 2015; Contarini et al., 2017; Liu et al., 2017; Valle et al., 2018). Currently, detailed studies and comparisons on the nutrient composition of bovine milk in different lactation stages have been more prevalent (Yang et al., 2016, 2017; Cao et al., 2019). Relatively few studies have been done on the alterations of the nutrient composition and interactions of donkey milk during the stages of lactation using high-throughput methodologies. We aimed to address this by using metabolomics to study the alterations in the composition of donkey milk during different lactation periods.

Metabolomics is a new branch of "-omics" science that studies the composition, relative abundance, dynamics, and interactions among metabolites in a given organism, and the biological systems in response to various stimuli or interventions (Osorio et al., 2012). Previous studies on milk metabolomics have focused on the comparison of the milk components from different species and their physiological roles (Scano et al., 2014, 2016; Dessi et al., 2016), or the comparison of milk metabolites under different physiological conditions (Tian et al., 2016; Xi et al., 2017). These studies confirmed the reliability and prospects of metabolomics in dairy research. Therefore, untargeted metabolomics based on 
ultra-high-performance liquid tandem chromatography quadrupole time-of-flight mass spectrometry (UHPLC-QTOF-MS) were chosen for this investigation.

In this study, using an UHPLC-QTOF-MS untargeted metabolomics approach, we aimed to comprehensively characterize the metabolites in donkey colostrum (DC) and mature milk (DMM), investigate their interactions and metabolic pathways, and further explore the composition alteration mechanisms in donkey milk during lactation. Our data provide practical information for the development of donkey milk products and a foundation for future research on specific milk nutrients.

\section{MATERIALS AND METHODS}

\section{Milk Sample Collection}

Fresh donkey milk samples, including colostrum (0-5 d postpartum) and mature milk (1-6 mo postpartum), were collected from 120 Chinese Dezhou donkeys on a local farm in Dalian, China. For each milk sample type (colostrum and mature milk), 60 samples were randomly divided into 6 groups (10 samples were pooled for each group), and 6 groups of each milk type were then tested and analyzed. The inclusion criteria of this study were as follows: healthy Chinese Dezhou donkeys aged between 2 and 4 yr, weighing $280 \pm 30 \mathrm{~kg}$ (mean \pm SEM), firstborn, fed the same amount (alfalfa as main feed), and living in same environment. The daily milk yield of each donkey was $1.0 \pm 0.3 \mathrm{~kg}$ (mean \pm SEM). All samples were transported to the laboratory in an icebox and stored at $-80^{\circ} \mathrm{C}$, until analysis. The collection of milk samples was approved by Shenyang Agricultural University (permit number: 2018-966) and was conducted in accordance with the practices outlined in the Guide for the care and use of agricultural animals in research and teaching (FASS, 2010).

\section{Chemicals and Quality Control Samples}

The chemicals used in this study were as follows: methanol (67-56-1, CNW Technologies, Düsseldorf, Germany), acetonitrile (75-05-8, CNW Technologies), ammonium acetate (631-61-8, CNW Technologies), ammonium hydroxide (1336-21-6, CNW Technologies), and 2-chloro-L-phenylalanine (103616-89-3, Shanghai Hengbai Biological Technology Co., Ltd., Shanghai, China). Quality control (QC) samples were equally mixed from metabolite extracts of each sample from the 12 groups, including $6 \mathrm{DC}$ and 6 DMM groups. Three identical QC samples were used to identify metabolites in the donkey milk and to ensure the reliability of the experimental results.

\section{Sample Extracts}

After being thawed at $4^{\circ} \mathrm{C}, 100 \mu \mathrm{L}$ of each sample was placed in an Eppendorf tube, then extracted with $400 \mu \mathrm{L}$ of extraction mixture (methanol: acetonitrile $1: 1 \mathrm{vol} / \mathrm{vol}$ ), followed by a vortex for $30 \mathrm{~s}$, then ultrasound treatment for $10 \mathrm{~min}$ (incubated in ice water), and incubation for $1 \mathrm{~h}$ at $-20^{\circ} \mathrm{C}$ to precipitate the proteins. Samples were then centrifuged at $13,300 \times$ $g$ for $15 \mathrm{~min}$ at $4^{\circ} \mathrm{C}$, and the supernatant $(450 \mu \mathrm{L})$ was transferred to fresh Eppendorf tubes and dried in a vacuum concentrator without heating. Extraction mixture $[100 \mu \mathrm{L}$ (acetonitrile:water 1:1 vol/vol)] was added to reconstitute the samples. They were then vortexed for $30 \mathrm{~s}$, sonicated for $10 \mathrm{~min}$ in a $4^{\circ} \mathrm{C}$ water bath, centrifuged for $15 \mathrm{~min}$ at $13,300 \times g$ and $4^{\circ} \mathrm{C}$, and finally the supernatant $(60 \mu \mathrm{L})$ was transferred into a 2 $\mathrm{mL}$ of a liquid chromatography (LC)/MS glass vial for UHPLC-QTOF-MS analysis.

\section{LC-MS/MS Analysis}

The LC-MS/MS analyses were performed using a UHPLC system (1290, Agilent Technologies, Santa Clara, CA) with a UPLC BEH amide column $(1.7 \mu \mathrm{m}$ $2.1 \times 100 \mathrm{~mm}$, Waters, Milford, MA) coupled to TripleTOF 5600 (Q-TOF, AB Sciex, Concord, ON, Canada). The mobile phase consisted of $25 \mathrm{mM} \mathrm{NH}_{4} \mathrm{OAc}$ and 25 $\mathrm{mM} \mathrm{NH}_{4} \mathrm{OH}$ in water $(\mathrm{pH}=9.75 ; \mathrm{A})$ and acetonitrile (B) was carried with the elution gradient as follows: 0 min, 95\% B; 7 min, 65\% B; 9 min, 40\% B; 9.1 min, $95 \%$ B; $12 \mathrm{~min}, 95 \% \mathrm{~B}$, which was delivered at $0.5 \mathrm{~mL} / \mathrm{min}$. The injection volume was $3 \mu \mathrm{L}$. The Triple TOF mass spectrometer was used for its ability to acquire MS/ MS spectra on an information-dependent basis during an LC/MS experiment. In this mode, the acquisition software (Analyst TF 1.7, AB Sciex) continuously evaluates the full scan survey MS data as it collects and triggers the acquisition of MS/MS spectra, depending on preselected criteria. In each cycle, 12 precursor ions whose intensities were greater than 100 were chosen for fragmentation at collision energy of $30 \mathrm{~V}$ (15 MS/ MS events with product ion accumulation time of 50 ms each). The electrospray ionization source conditions were set as follows: ion source gas 1 as $60 \mathrm{~Pa}$, ion source gas 2 as $60 \mathrm{~Pa}$, curtain gas as $35 \mathrm{~Pa}$, source temperature $650^{\circ} \mathrm{C}$, ion spray voltage floating 5,000 or $-4,000 \mathrm{~V}$ in the positive or negative modes, respectively.

\section{Data Processing}

The MS raw data (.wiff) files were converted to the mzXML format using ProteoWizard, and processed by 
R package XCMS (version 3.2; Smith et al., 2006). The preprocessing results generated a data matrix that consisted of the retention time, mass to-charge ratio $(\mathrm{m} / \mathrm{z})$ values, and peak intensity. The $\mathrm{R}$ package CAMERA was used for peak annotation after XCMS data processing (Kuhl et al., 2012). An in-house MS2 database was applied for metabolite identification. Additionally, the MS raw data were preprocessed to remove the adverse effects caused by the outliers. First, the missing values were filled by half of the minimum positive value in the MS raw data. Then, the normalization and log conversion were performed before analysis.

\section{Bioinformatics Analysis}

The data were then processed by SIMCA 15 software package (Umetrics, Umea, Sweden) for principal component analysis, orthogonal projections to latent structure-discriminate analysis (OPLS-DA), and permutation tests of OPLS-DA. The correlation network analysis was performed using the Cytoscape 3.7.0 software package (Seattle, WA; Doerks et al., 2002). The hierarchical clustering analysis and metabolism pathways plot were performed using the web-based MetaboAnalyst (http://www.metaboanalyst.ca; Chong et al., 2018; Chong and Xia, 2018). The Venn diagram was constructed using the program "Calculate and draw custom Venn diagrams online" (http://bioinformatics .psb.ugent.be). In addition, commercial databases, including Kyoto Encyclopedia of Genes and Genomes (KEGG) pathway database (https://www.kegg.jp/ kegg/) and Human Metabolome Database (http://www .hmdb.ca), were used for qualitative analysis and to search for metabolites in the biosynthesis pathway.

\section{RESULTS}

\section{Metabolite Profiles of DC and DMM}

Compared with the positive ion mode, more metabolites were detected in the negative ion mode and this was therefore used for later analysis. A total of 1,725 peaks were detected for both the DC and DMM; after utilizing the interquartile range de-noising method, 270 metabolites remained (Supplemental Table S1, https:// doi.org/10.3168/jds.2019-17448). Half of the minimum value was used for the missing values in the raw data. Additionally, the internal standard normalization method was employed in the data analysis. According to the Leco/Fiehn Metabolomics Library (Leco Corporation, St. Joseph, MI), the majority of metabolites were endogenous, including AA, carboxylic acids, fatty acids, lipids, nucleosides, saccharides, and their derivatives. It should be noted that the value for the metabolites in Supplemental Table S1 (https://doi.org/10.3168/jds .2019-17448) is not the concentration, but the response value of the metabolite. The magnitude of the response value represents the response of the substance to the instrument. Here, the size of the response value was used to represent the relative content of the metabolite.

\section{Statistical Comparison of Metabolites Between DC and DMM}

As shown in Figure 1a, the principal component analysis plot showed the distribution of the MS raw data, which demonstrated that there were separations between the DC and DMM groups. To better understand the variables responsible for the classification and achieve a higher level of group separation, supervised OPLS-DA was adopted (Figure 1b). The parameters for the classifications were $R^{2} Y=0.995$ and $Q^{2} Y=$ 0.966 , which indicated good classification predictive ability and stability of the model. The permutation test was performed to further validate the model and to estimate its robustness and predictive power (Figure 1c). The results demonstrated the stability of the model, its low risk for over-fitting, and its reliability. Additionally, a loading plot was constructed based on OPLS-DA (Figure 1d) to better visualize the important variables that were situated far from the origin and their contribution to the differences between the DC and DMM groups.

\section{Identification of Significantly Different Metabolites Between DC and DMM}

To understand the alterations of the metabolites in the donkey milk during lactation, and provide data for nutritional research, metabolites that were significantly different between the DC and DMM groups were screened. As shown in the Venn diagram (Figure 2a), the variable importance in the projection (VIP) values exceeding 1.20 were first selected (Figure $2 \mathrm{~b}$ ). Then the remaining variables were assessed by Student's $t$-test $(P<0.05)$. Fold change $(\mathbf{F C})$ value $(\mathrm{DC}$ vs. DMM $)$ was also used to further narrow the threshold of the significantly different metabolites (SDM; FC $>1.20$ or $<0.83)$. A total of $52 \mathrm{SDM}$ were finally selected between the DC and DMM groups (Figure 2c). Among them, the levels of 8 metabolites were found to be significantly downregulated and 44 were upregulated in the DC group compared with the DMM group.

\section{Correlation Network Analysis of the SDM}

To identify the interactions of the SDM with similar physiological and molecular characteristics, a correla- 
tion network analysis was performed, and showed that some SDM were highly correlated (Figure 3). Twelve clusters were formed according to their chemical structures and similarity of physiological functions, revealing the alterations in donkey milk metabolite homeostasis during lactation. The correlation within lipids (25 edges) was found to be the strongest, and these lipids had no correlation with the other SDM. Furthermore, the changes in the concentration of one SDM may cause changes in the other SDM, but the trend was not always consistent.

\section{Key Differential Metabolic Pathways Between DC and DMM}

To understand the alterations in the composition of donkey milk during lactation, and to explore the mechanisms changing the metabolite content during lactation,
52 SDM and their concentration changes were mapped to KEGG, Human Metabolome Database, and PubChem databases. The SDM were found to be involved in 32 metabolic pathways. Based on their impact value to each metabolic pathway and $P$-value, 11 key differential metabolic pathways that might cause changes during the lactation of donkey milk metabolites, involved in taurine and hypotaurine metabolism, lysine biosynthesis, purine metabolism, galactose metabolism, valine, leucine and isoleucine degradation, ascorbate and aldarate metabolism, glycosylphosphatidylinositol-anchor biosynthesis, glycerophospholipid metabolism, histidine metabolism, fatty acid elongation in mitochondria, and the citrate cycle [tricarboxylic acid (TCA) cycle], were screened out (Figure 4). Based on the 11 key differential metabolic pathways, as well as the identified SDM and their changes between DC and DMM, a hypothesized scheme pathway related to the metabolite changes of a
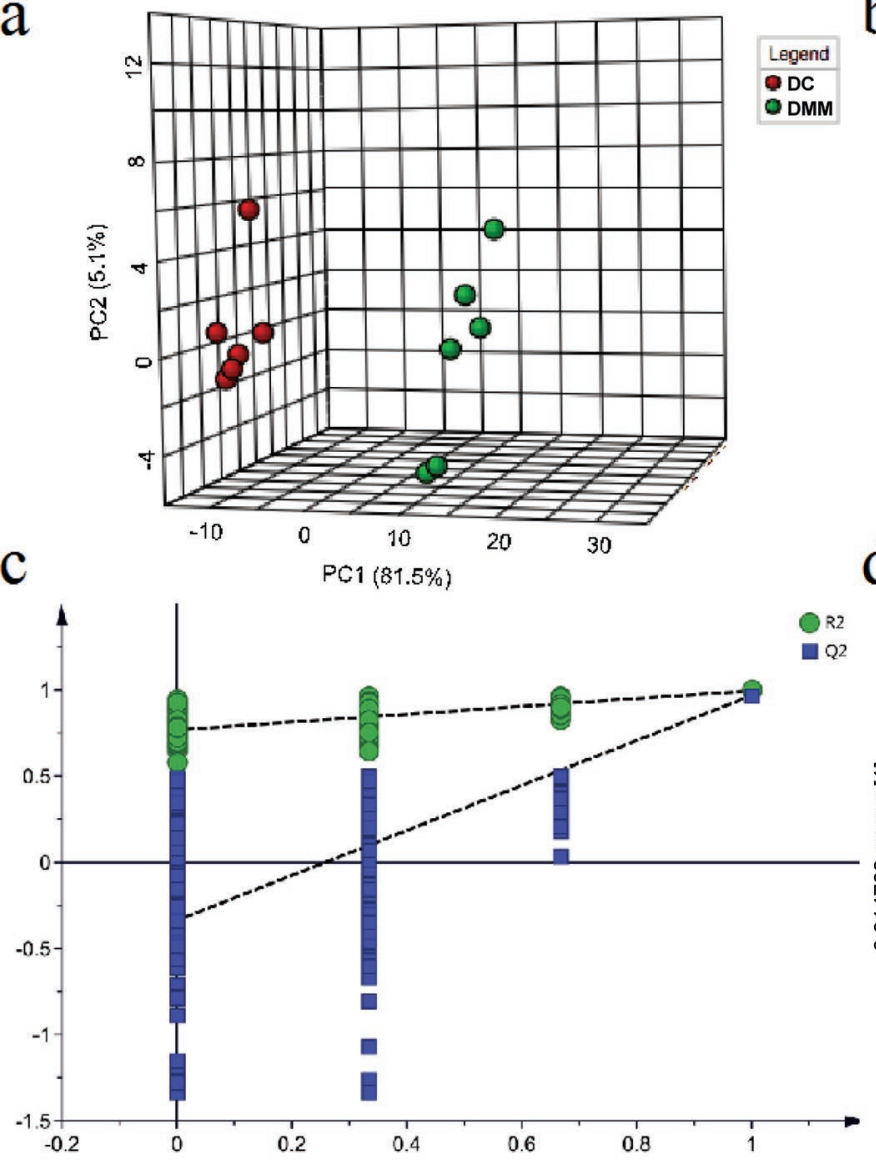
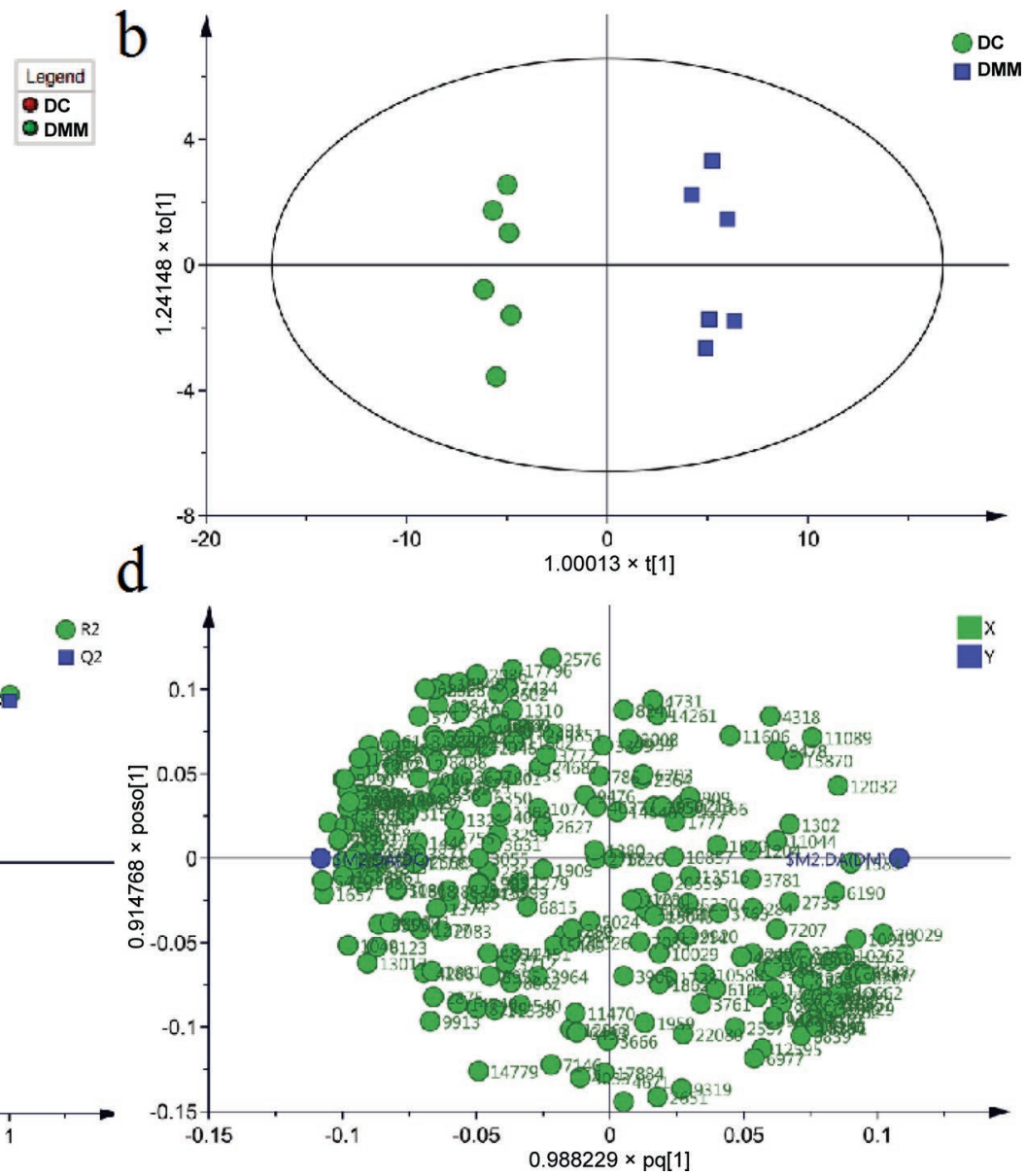

Figure 1. Principal component (PC) analysis score plot (a), orthogonal projections to latent structures-discriminate analysis (OPLS-DA) score plot (b), corresponding validation plot of OPLS-DA (c), and loading plot of 270 identified metabolites in donkey colostrum (DC) and mature milk $(\mathrm{DMM} ; \mathrm{d}) . \mathrm{R} 2=$ fraction of the $\mathrm{Y}$ variation modeled in the component, using the $\mathrm{Y}$ model; $\mathrm{Q} 2=$ fraction of $\mathrm{Y}$ variation predicted according to cross-validation in the component, using the $\mathrm{X}$ model; poso[1] = orthogonal loading po of the X-part and the projection of to onto $\mathrm{Y}$, so, combined to one vector. Available for OPLS and O2PLS; pq[1] = X loading weight $\mathrm{p}$ and $\mathrm{Y}$ loading weight $\mathrm{q}$ combined to one vector. Available for OPLS and O2PLS. 
a

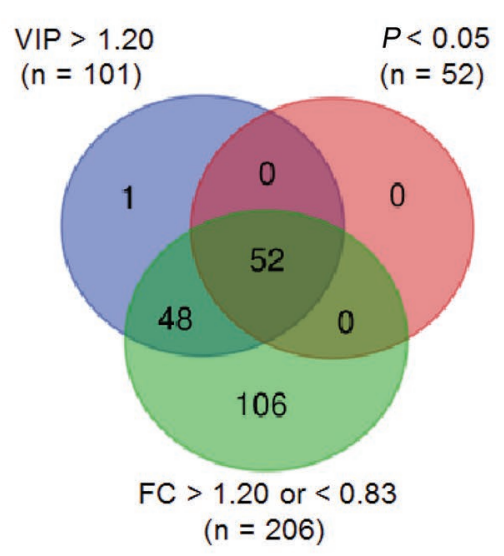

b

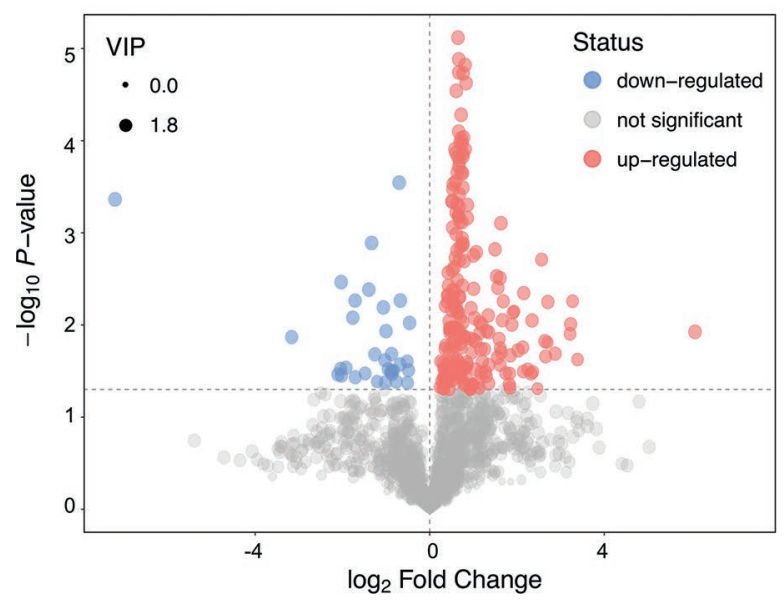

c

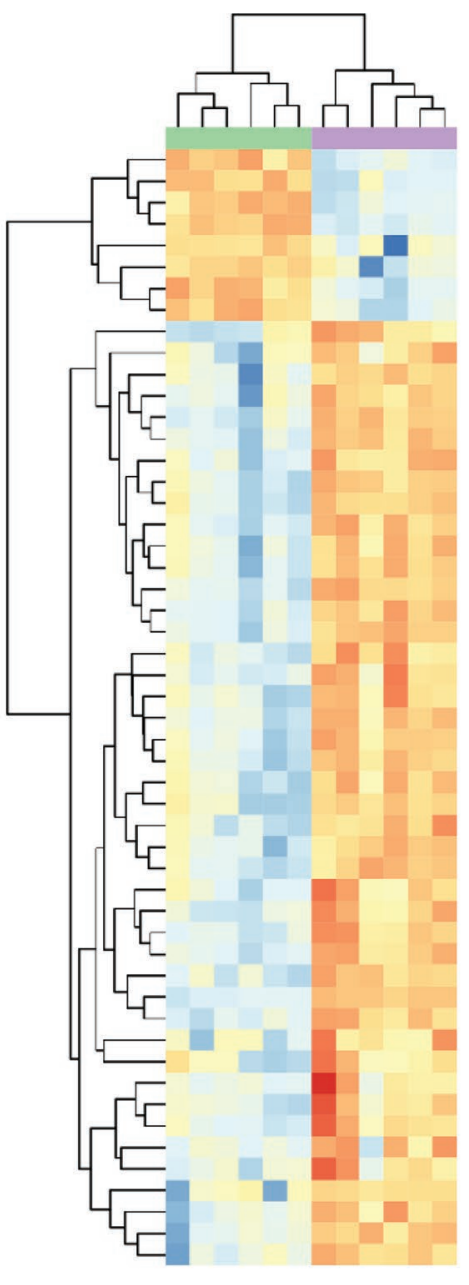

Sterol-3-beta-D-glucoside 1,3-Dipropyl-8-cyclopentylxanthine Pentadecanoic Ácid Raffinose

Lacto-N-neohexaose Nicotinami glucuronide Pyrimidine PA(34:1) 2-Furoic acid Xanthylic acid L-Cysteinesulfinic-acid PE(29:1) D-Arabinono-1-4-lactone TG(48:3) Nicotinuric acid L-Histidine Dihydrouracil PS (36:6)

Hypotaurin

Folic acid

Sialyllactose

Phenylacetylglycine

1-Methyluric-acid

Hippuric acid

ketoisocaproic acid Caffeic Acid Gibberellin Aspirin

Lex-lactose

Dioxybenzone

N.Butyrylglycine

3-Oxotetradecanoyl-CoA
PE $(30: 0)$

2-Oxoadipic acid

PE(36:4)

Taurine

m-Chlorohippuric acid Quercetin

Glycerophosphocholine

PE(48:5)

Dulcito

D-Quinovose

cis-Aconitate

Mesaconic acid 6 -

2-Deoxy-D-glu

Ascorbic a
DG $(46: 2)$

Adenosine-monophosphate

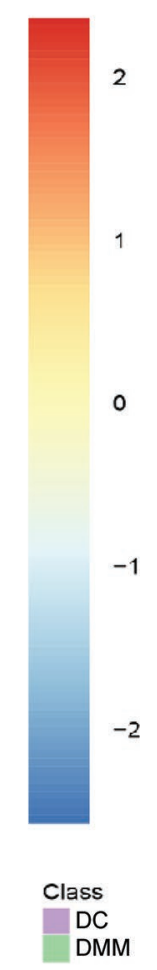

Figure 2. Venn diagram (a) of variable importance in the projection (VIP), $P$-value, and fold change (FC) results, and volcano plot (b) of the metabolites in donkey colostrum (DC) and mature milk (DMM). Heat map analysis of 52 significantly different metabolites between DC and DMM (c). PA = glycerophosphatidic acid; PE = glycerophosphatidylethanolamine; PS = glycerophosphatidylserine; TG = triacylglycerol; $\mathrm{DG}=$ diacylglycerol.

the donkey milk during lactation was integrated and visualized, including the metabolism of some carboxylic acids, AA, and fatty acids (Figure 5).

\section{DISCUSSION}

Previously, using a GC-MS method, Murgia et al. explored the composition characteristics of donkey milk and identified 45 metabolites (Murgia et al., 2016). Herein, using UHPLC-QTOF-MS-based untargeted metabolomics technology, 1,725 peaks were detected and then 270 metabolites were finally characterized in donkey milk. Some of the metabolites identified, such as uridine and thymidine, had not previously been described in donkey milk. Studies have shown that human milk is rich in a variety of nucleotides including uridine, which accounts for 2 to $5 \%$ of the NPN in human milk
(German et al., 2002; Xiao et al., 2014). It has been demonstrated that the nucleotides in human milk play a significant role in improvement of gastrointestinal tract repair after damage, enhancement of the metabolism of fatty acids, and immune system functions (Carver and Walker, 1995; Schlimme et al., 2000). In this study, uridine was identified in both DC and DMM, thereby providing useful information for nutritional database information and the QC of nucleotides in donkey milk. Myo-inositol, as a conditionally essential nutrient, has been shown to have chemopreventive and chemotherapeutic properties in cancer, cataracts, neuropathy, and nephropathy (Grases et al., 2002; Rizvi et al., 2006; Liu et al., 2009; Schlemmer et al., 2009; Corrado et al., 2011). Additionally, myo-inositol is a compound necessary for babies, and when this substance cannot be administered through breast milk, it must be sup- 
plied in the diet by inositol-enriched milk or formula (Hallman et al., 1987; Flores et al., 2011; Woollard et al., 2014; Indyk et al., 2016). In this study, myo-inositol was also identified in donkey milk. It can be seen from Supplemental Table S1 (https://doi.org/10.3168/jds .2019-17448) that the relative content of the myo-inositol was high in both the DC and DMM groups (0.379 in mean DC and 0.273 in mean DMM), and showed no significant differences (VIP $=0.832, P=0.358$, $\mathrm{FC}=1.391)$, which demonstrated the importance of myo-inositol in mammalian milk and its significance in the growth and development of mammalian cubs. By characterizing more metabolites in donkey milk, we deepen our understanding of its composition, supporting its improved use and the production of different types of formula milk powder to meet the needs of different populations.

The alterations in the composition of donkey milk metabolites during lactation periods were also explored. Our results indicated differences in the donkey milk metabolites as the lactation periods changed. Based on the combined results of the univariate and multivariate statistical analysis, 52 SDM were screened out, including 8 downregulated metabolites and 44 upregulated metabolites in the DC group, compared with the DMM group. Previous studies have proven that the lipid content in donkey milk is different during the lactation period (Martemucci and D'Alessandro, 2012; Martini et al., 2015), and our research confirmed this. It can be seen from Figure 2 that the levels of 8 lipids are upregulated in DC compared with DMM, including 2 glycerolipids [triglycerides (48:3) and diglycerides (46:2)] and 6 glycerophospholipids [glycerophosphatidic acid (34:1), glycerophosphatidylethanolamine (PE; 29:1), PE (30:0), PE (36:4), PE (48:5), glycerophosphatidylserine (36:6)]. The $\mathrm{PE}$ is one of the most important components of glycerophospholipids in milk because it plays an important role in biological processes such as cell signaling and apoptosis, and it is also a component of mammalian cell membranes (Vance, 2008). In addition, PE has shown the ability to enhance the development of brain functions and improve memory (Modica-Napolitano and Renshaw, 2004). Thus, the upregulated level of PE in DC might be closely related to the development of the nervous system of donkey foals. However, the specific physiological functions and roles of PE in DC require further study. The screened SDM are helpful to study the physiological functions of donkey milk at different lactation stages and to explore the targets related to growth and development, and

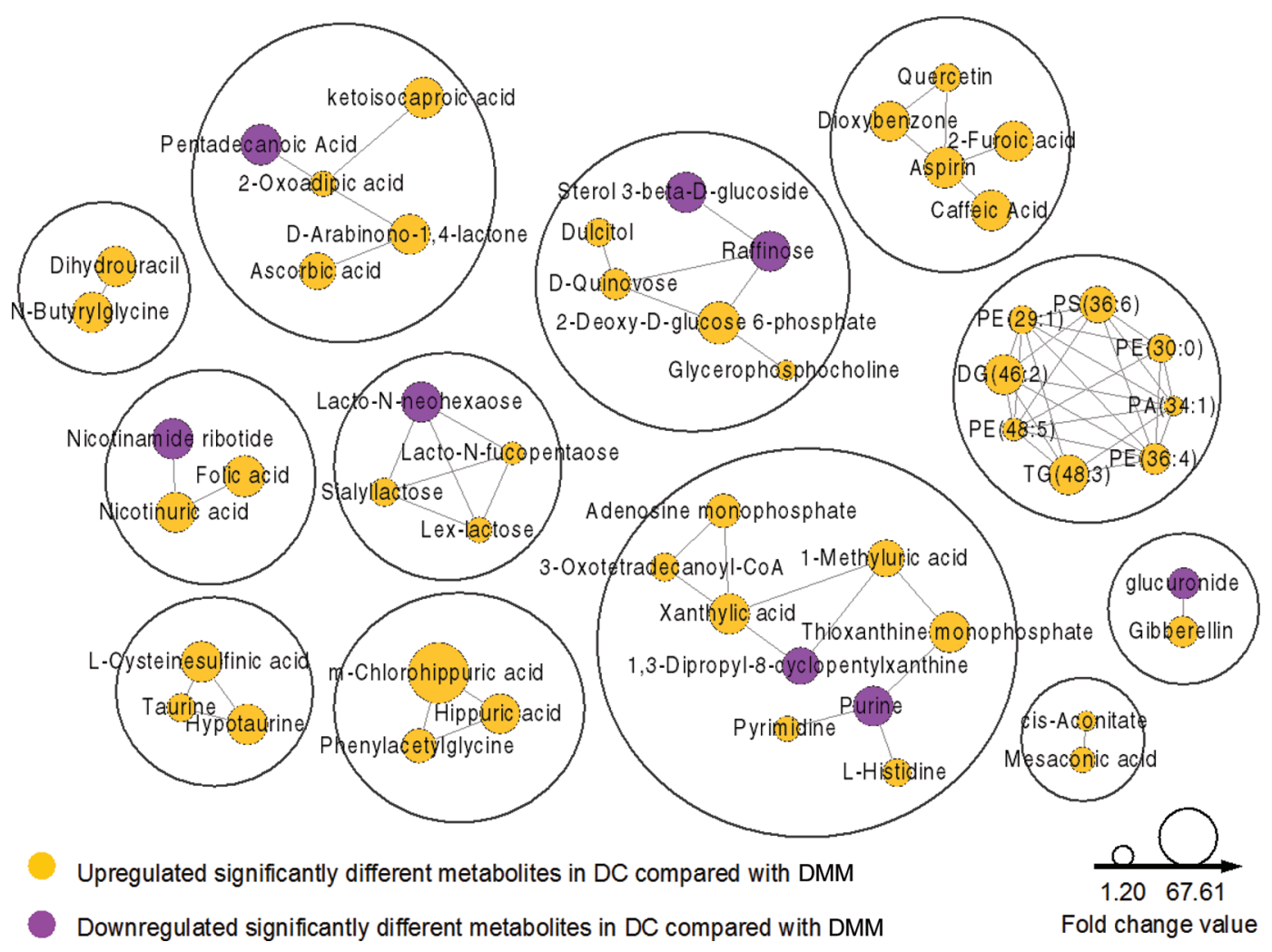

Figure 3. Correlation network of 52 significantly different metabolites in donkey colostrum (DC) and mature milk (DMM). PA $=$ glycerophosphatidic acid; PE = glycerophosphatidylethanolamine; PS = glycerophosphatidylserine; $\mathrm{TG}=$ triacylglycerol; $\mathrm{DG}=$ diacylglycerol. 
they provide direction to further study the significance of specific metabolites to pups and babies under different lactation stages.

After screening 52 SDM, the correlation network analysis was performed based on the similarity of chemical structures and physiological functions. Twelve clusters were formed, of which the highest connectivity was a cluster of 6 lipids, which also verified the effect of the lactation on donkey milk lipids. In addition, the largest number of nodes for these clusters contained 9 SDM. This may be related to their physiological function and metabolism. Both upregulated and downregulated metabolites are present in the 9 SDM, which indicates that the alterations of metabolites in the donkey milk with lactation are complex. Changing of one metabolite may cause the change of another metabolite, but the trend is not always consistent. To further elucidate the interrelationship between the $52 \mathrm{SDM}$ and their physiological effects, 11 key differential metabolic pathways were found that might cause alterations in donkey milk metabolites during lactation (Figure 4). These biochemical alterations might be used to understand the effects of the different lactation periods on donkey milk composition and aid in the future exploration of the nutrition of donkey milk.

The hypothesized scheme pathways most related to the metabolite changes of donkey milk during lactation are manually linked together based on the significantly changed pathways from the different metabolites, and the results of key different metabolic pathways. The hypothesized scheme pathway integrated by key differential metabolic pathways mainly included the TCA cycle; taurine and hypotaurine metabolism; valine, leucine, and isoleucine degradation; fatty acid metabolism; fatty acid elongation in mitochondria; and ascorbate and aldarate metabolism. It can be seen from Figure 5 that these metabolic pathways are mainly connected and transmitted by acetyl-CoA in the TCA cycle as a bridge. Therefore, we hypothesize that the changes of taurine and hypotaurine metabolism and valine, leucine, and isoleucine degradation might affect the level of acetyl-CoA, which might lead to the changes of fatty

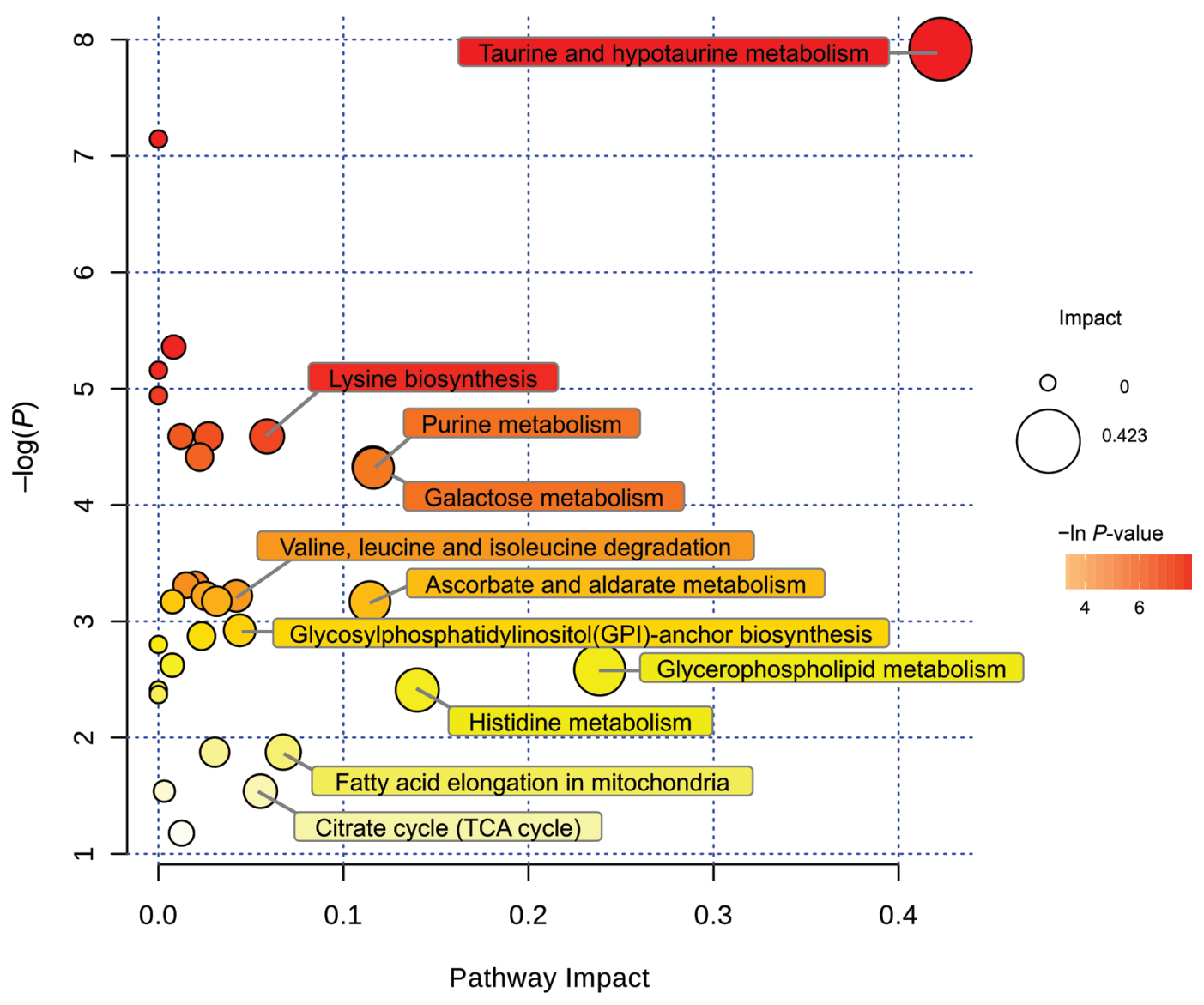

Figure 4. Metabolomic view map of the 52 significantly different metabolites of biosynthetic pathways between donkey colostrum (DC) and mature milk (DMM). The $\mathrm{x}$-axis represents the pathway effect, and the $\mathrm{y}$-axis represents the $-\ln P$-value. Large sizes and dark colors represent the major pathway enrichment and high pathway effect values, respectively. TCA = tricarboxylic acid. 


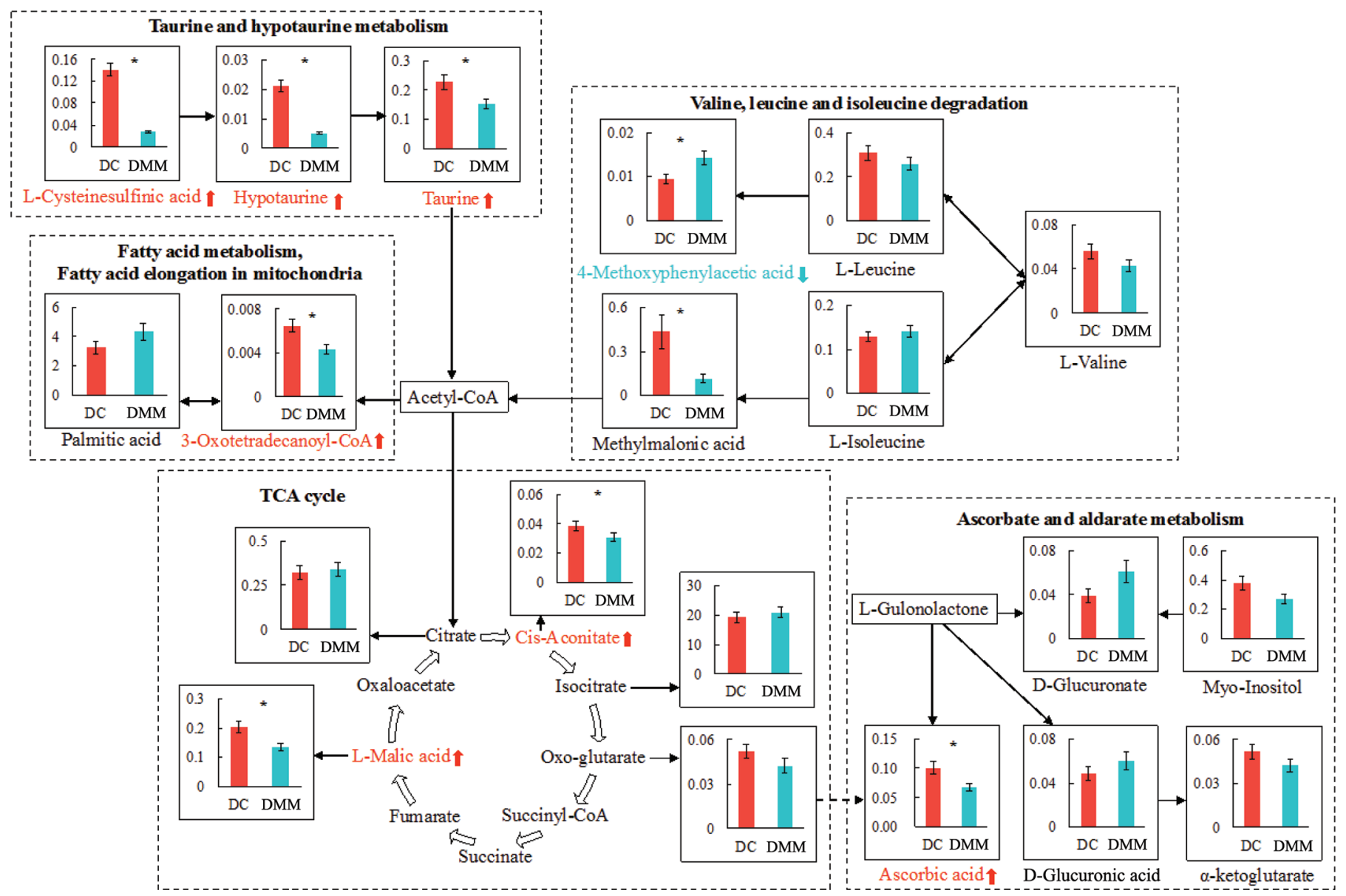

Figure 5. Hypothesized scheme pathway most related to metabolite changes of donkey milk during lactation. DC = donkey colostrum; $\mathrm{DMM}=$ donkey mature milk; TCA = tricarboxylic acid. Error bars indicate SEM. *Asterisks indicate metabolites with significant differences $(P<0.05)$.

acid metabolism, and fatty acid elongation in the mitochondria and the TCA cycle, and subsequently to the alterations of ascorbic acid metabolism. Previous studies have found that branched AA, including isoleucine and valine, play a significant role in regulating protein conversion (Cappello et al., 2017), and also promote energy metabolism by entering gluconeogenesis and enlarging the pool of TCA cycle intermediates (Shimomura et al., 2004; Wagner et al., 2014). In this study, the level of methylmalonic acid is higher in DC than DMM. Additionally, both 4-methoxyphenylacetic acid and methylmalonic acid are metabolites of L-valine. The decrease of the level of 4-methoxyphenylacetic acid also proves that the upregulation of methylmalonic acid contents, which indicates that isoleucine and valine promote the production of metabolites in the TCA cycle. Therefore, the high level of isoleucine, valine, and their metabolites might play a role in promoting the rapid growth and development of mammalian pups in the primary stage. Taurine has been shown to be in- volved in the basic processes of brain cell development (Pasantes-Morales and Hernandez-Benitez, 2010) and is the highest free AA present in neonatal and developing brains, being 3 to 4 times higher than that in adults. This decline is a consistent feature observed among species, regardless of their differences in taurine concentration (Sturman, 1993; Miller et al., 2000). These results indicate a requirement for taurine when brain cells are in the process of maturation. In addition, studies have shown that the concentration of taurine is the highest of the free AA in milk, including human milk, bovine milk, and donkey milk (Rassin et al., 1978; Guo et al., 2007). In our results of taurine and hypotaurine metabolism, the levels of L-cysteinesulfinic acid, hypotaurine, and taurine are all significantly upregulated in DC than in DMM $(P<0.05)$, thereby affecting the level of acetyl-CoA based on the KEGG pathway, and possibly further affecting the development of the brain and nervous system of donkey foals. A previous study has shown that ascorbic acid is able to enhance ferritin 
mRNA translation by an IRP/aconitase switch, thus indicating a link between ascorbic acid and aconitate (Toth and Bridges, 1995). In this study, the levels of cis-aconitate and L-malic acid in the TCA cycle are significantly higher in DC compared with DMM; meanwhile, the level of ascorbic acid in the ascorbate and aldarate metabolism is significantly higher in DC $(P$ $<0.05)$. By integrating key metabolic pathways into a hypothesized scheme pathway, we deepened our understanding of the metabolic mechanisms of donkey milk during lactation and have provided a forward-looking perspective for future research and development of donkey milk to meet human nutritional requirements.

\section{CONCLUSIONS}

In the current study, metabolites in donkey milk between the different lactation periods were analyzed using an untargeted metabolomics approach. A total of 270 metabolites were identified in both DC and DMM. We focused on the 52 SDM that were significantly differentially regulated between the milk types and investigated them using bioinformatics analysis methods. The results from the present study provide further insights into the mechanisms, at a metabolomics level, that can partially explain the alterations of donkey milk between lactation, providing an in-depth understanding of the potential mechanisms of donkey milk lactation. The identified SDM and related metabolism pathways may serve as potential biomarkers, which might provide practical information for the development of donkey milk products.

\section{ACKNOWLEDGMENTS}

This work was supported by the by National Key R \& D Program of China (grant number: 2018YFC1604302), "Twelfth Five Year" National Science and Technology Plan Project (grant number: 2013BAD18B03, China), and Shenyang Technological Innovation Project (grant number: Y17-0-028, China). We thank the Dalian "Shenghongdaoda" and Tieling "Xingfazhongchu" farms for providing donkey milk samples.

\section{REFERENCES}

Cao, X., S. Kang, M. Yang, W. Li, S. Wu, H. Han, L. Meng, R. Wu, and X. Yue. 2018. Quantitative N-glycoproteomics of milk fat globule membrane in human colostrum and mature milk reveals changes in protein glycosylation during lactation. Food Funct. 9:1163-1172.

Cao, X., M. Yang, N. Yang, X. Liang, D. Tao, B. Liu, J. Wu, and X. Yue. 2019. Characterization and comparison of whey N-glycoproteomes from human and bovine colostrum and mature milk. Food Chem. 276:266-273.
Cappello, T., M. Maisano, A. Mauceri, and S. Fasulo. 2017. (1)H NMRbased metabolomics investigation on the effects of petrochemical contamination in posterior adductor muscles of caged mussel Mytilus galloprovincialis. Ecotoxicol. Environ. Saf. 142:417-422.

Carver, J. D., and W. A. Walker. 1995. The role of nucleotides in human nutrition. J. Nutr. Biochem. 6:58-72.

Chiofalo, B., P. Dugo, I. L. Bonaccorsi, and L. Mondello. 2011. Comparison of major lipid components in human and donkey milk: New perspectives for a hypoallergenic diet in humans. Immunopharmacol. Immunotoxicol. 33:633-644.

Chong, J., O. Soufan, C. Li, I. Caraus, S. Li, G. Bourque, D. S. Wishart, and J. Xia. 2018. MetaboAnalyst 4.0: Towards more transparent and integrative metabolomics analysis. Nucleic Acids Research 46(Web Server issue):W486-W494.

Chong, J., and J. Xia. 2018. MetaboAnalystR: An R package for flexible and reproducible analysis of metabolomics data. Bioinformatics 34:4313-4314

Contarini, G., V. Pelizzola, S. Scurati, and M. Povolo. 2017. Polar lipid of donkey milk fat: Phospholipid, ceramide and cholesterol composition. J. Food Compos. Anal. 57:16-23.

Corrado, F., R. D'Anna, V. G. Di, D. Giordano, B. Pintaudi, A. Santamaria, and B. A. Di. 2011. The effect of myoinositol supplementation on insulin resistance in patients with gestational diabetes. Diabet. Med. 28:972-975.

Dessi, A., A. Murgia, R. Agostino, M. G. Pattumelli, A. Schirru, P. Scano, V. Fanos, and P. Caboni. 2016. Exploring the role of different neonatal nutrition regimens during the first week of life by urinary GC-MS metabolomics. Int. J. Mol. Sci. 17:265.

Doerks, T., R. R. Copley, J. Schultz, C. P. Ponting, and P. Bork. 2002 Systematic identification of novel protein domain families associated with nuclear functions. Genome Res. 12:47-56.

FASS. 2010. Guide for the care and use of agricultural animals in research and teaching. 3rd ed. FASS Inc., Champaign, IL.

Flores, M. I., J. L. Moreno, A. G. Frenich, and J. L. Vidal. 2011. Fast determination of myo-inositol in milk powder by ultra high performance liquid chromatography coupled to tandem mass spectrometry. Food Chem. 129:1281-1286.

German, J. B., G. C. J. Dillard, and R. E. Ward. 2002. Bioactive components in milk. Curr. Opin. Clin. Nutr. Metab. Care 5:653-658.

Grases, F., B. M. Simonet, I. Vucenik, J. Perelló, R. M. Prieto, and A. K. M. Shamsuddin. 2002. Effects of exogenous inositol hexakisphosphate (Ins P 6) on the levels of Ins P 6 and of inositol trisphosphate (Ins P 3) in malignant cells, tissues and biological fluids. Life Sci. 71:1535-1546.

Guo, M. R., P. H. Dixon, Y. W. Park, J. A. Gilmore, and P. S. Kindstedt. 2001. Seasonal changes in the chemical composition of commingled goat milk. J. Dairy Sci. 84(E. Suppl.):E79-E83.

Guo, H. Y., K. Pang, X. Y. Zhang, L. Zhao, S. W. Chen, M. L. Dong, and F. Z. Ren. 2007. Composition, physiochemical properties, nitrogen fraction distribution, and amino acid profile of donkey milk. J. Dairy Sci. 90:1635-1643.

Hallman, M., P. Arjomaa, and K. Hoppu. 1987. Inositol supplementation in respiratory distress syndrome: Relationship between serum concentration, renal excretion, and lung effluent phospholipids. J. Pediatr. 110:604-610.

Indyk, H. E., S. C. Saldo, P. M. White, M. N. Dole, B. D. Gill, and D. C. Woollard. 2016. The free and total myo-inositol contents of early lactation and seasonal bovine milk. Int. Dairy J. 56:33-37.

Kuhl, C., R. Tautenhahn, C. Böttcher, T. R. Larson, and S. Neumann. 2012. CAMERA: An integrated strategy for compound spectra extraction and annotation of liquid chromatography/mass spectrometry data sets. Anal. Chem. 84:283-289.

Liu, Z., A. Logan, B. G. Cocks, and S. Rochfort. 2017. Seasonal variation of polar lipid content in bovine milk. Food Chem. 237:865-869.

Liu, X., P. W. Villalta, and S. J. Sturla. 2009. Simultaneous determination of inositol and inositol phosphates in complex biological matrices: Quantitative ion-exchange chromatography/tandem mass spectrometry. Rapid Commun. Mass Spectrom. 23:705-712.

Mao, X., J. Gu, Y. Sun, S. Xu, X. Zhang, H. Yang, and F. Ren. 2009 Anti-proliferative and anti-tumour effect of active components 
in donkey milk on A549 human lung cancer cells. Int. Dairy J. 19:703-708.

Martemucci, G., and A. G. D'Alessandro. 2012. Fat content, energy value and fatty acid profile of donkey milk during lactation and implications for human nutrition. Lipids Health Dis. 11:113.

Martini, M., I. Altomonte, E. Manica, and F. Salari. 2015. Changes in donkey milk lipids in relation to season and lactation. J. Food Comp. Anal. 41:30-34.

Miller, T. J., R. D. Hanson, and P. H. Yancey. 2000. Developmental changes in organic osmolytes in prenatal and postnatal rat tissues. Comp. Biochem. Physiol. A Mol. Integr. Physiol. 125:45-56.

Modica-Napolitano, J. S., and P. F. Renshaw. 2004. Ethanolamine and phosphoethanolamine inhibit mitochondrial function in vitro: Implications for mitochondrial dysfunction hypothesis in depression and bipolar disorder. Biol. Psychiatry 55:273-277.

Murgia, A., P. Scano, M. Contu, I. Ibba, M. Altea, M. Bussu, M. Demuru, A. Porcu, and P. Caboni. 2016. Characterization of donkey milk and metabolite profile comparison with human milk and formula milk. LWT 74:427-433.

Osorio, M. T., A. P. Moloney, L. Brennan, and F. J. Monahan. 2012. Authentication of beef production systems using a metabolomicbased approach. Animal 6:167-172.

Pasantes-Morales, H., and R. Hernandez-Benitez. 2010. Taurine and brain development: Trophic or cytoprotective actions? Neurochem. Res. 35:1939-1943.

Polidori, P., and S. Vincenzetti. 2013. Use of donkey milk in children with cow's milk protein allergy. Foods 2:151-159.

Rassin, D. K., J. A. Sturman, and G. E. Guall. 1978. Taurine and other free amino acids in milk of man and other mammals. Early Hum. Dev. 2:1-13.

Rizvi, I., D. R. Riggs, B. J. Jackson, A. Ng, C. Cunningham, and D. W. Mcfadden. 2006. Inositol hexaphosphate (IP6) inhibits cellular proliferation in melanoma. J. Surg. Res. 133:3-6.

Scano, P., A. Murgia, M. Demuru, R. Consonni, and P. Caboni. 2016. Metabolite profiles of formula milk compared to breast milk. Food Res. Int. 87:76-82.

Scano, P., A. Murgia, F. M. Pirisi, and P. Caboni. 2014. A gas chromatography-mass spectrometry-based metabolomic approach for the characterization of goat milk compared with cow milk. J. Dairy Sci. 97:6057-6066.

Schlemmer, U., W. Frølich, R. M. Prieto, and F. Grases. 2009. Phytate in foods and significance for humans: Food sources, intake, processing, bioavailability, protective role and analysis. Mol. Nutr. Food Res. 53(Suppl. 2):S330-75.

Schlimme, E., D. Martin, and H. Meisel. 2000. Nucleosides and nucleotides: Natural bioactive substances in milk and colostrum. Br. J. Nutr. 84(Suppl. 1):S59-S68.

Shimomura, T. M., N. Nakai, M. Nagasaki, and R. A. Harris. 2004. Exercise promotes BCAA catabolism: Effects of BCAA supplementation on skeletal muscle during exercise. J. Nutr. 134:1583S$1587 \mathrm{~S}$.

Smith, C. A., E. J. Want, O. M. Grace, A. Ruben, and S. Gary. 2006. XCMS: Processing mass spectrometry data for metabolite profiling using nonlinear peak alignment, matching, and identification. Anal. Chem. 78:779-787.
Sturman, J. A. 1993. Taurine in development. Physiol. Rev. 73:119147.

Tian, H., N. Zheng, W. Wang, J. Cheng, S. Li, Y. Zhang, and J. Wang. 2016. Integrated metabolomics study of the milk of heat-stressed lactating dairy cows. Sci. Rep. 6:24208.

Toth, I., and K. R. Bridges. 1995. Ascorbic acid enhances ferritin mRNA translation by an IRP/aconitase switch. J. Biol. Chem. 270:19540-19544

Valle, E., L. Pozzo, M. Giribaldi, D. Bergero, M. S. Gennero, D. Dezzutto, A. McLean, G. Borreani, M. Coppa, and L. Cavallarin 2018. Effect of farming system on donkey milk composition. J. Sci. Food Agric. 98:2801-2808

Vance, J. E. 2008. Thematic Review Series: Glycerolipids. Phosphatidylserine and phosphatidylethanolamine in mammalian cells: Two metabolically related aminophospholipids. J. Lipid Res. 49:13771387.

Wagner, L., S. Trattner, J. Pickova, P. Gomez-Requeni, and A. A. Moazzami. 2014. (1)H NMR-based metabolomics studies on the effect of sesamin in Atlantic salmon (Salmo salar). Food Chem. 147:98-105.

Woollard, D. C., C. Macfadzean, H. E. Indyk, A. Mcmahon, and S. Christiansen. 2014. Determination of myo-inositol in infant formulae and milk powders using capillary gas chromatography with flame ionisation detection. Int. Dairy J. 37:74-81.

Xi, X., L. Y. Kwok, Y. Wang, C. Ma, Z. Mi, and H. Zhang. 2017. Ultra-performance liquid chromatography-quadrupole-time of flight mass spectrometry MS(E)-based untargeted milk metabolomics in dairy cows with subclinical or clinical mastitis. J. Dairy Sci. 100:4884-4896.

Xiao, G., H. Xiao, Y. Zhu, and Y. You. 2014. Determination of nucleotides in Chinese human milk by high-performance liquid chromatography-tandem mass spectrometry. Dairy Sci. Technol. 94:591602.

Yang, M., X. Cao, R. Wu, B. Liu, W. Ye, X. Yue, and J. Wu. 2017. Comparative proteomic exploration of whey proteins in human and bovine colostrum and mature milk using iTRAQ-coupled LCMS/MS. Int. J. Food Sci. Nutr. 68:671-681.

Yang, M., M. Cong, X. Peng, J. Wu, R. Wu, B. Liu, W. Ye, and X. Yue. 2016. Quantitative proteomic analysis of milk fat globule membrane (MFGM) proteins in human and bovine colostrum and mature milk samples through iTRAQ labeling. Food Funct. $7: 2438-2450$.

Zhang, H., J. Y. D. Zhao, H. Liu, J. Li, and M. Guo. 2005. Changes in chemical composition of Alxa Bactrian camel milk during lactation. J. Dairy Sci. 88:3402-3410.

Zhang, X.-Y., L. Zhao, L. Jiang, M.-L. Dong, and F.-Z. Ren. 2008. The antimicrobial activity of donkey milk and its microflora changes during storage. Food Control 19:1191-1195.

\section{ORCIDS}

Mohan Li ๑ https://orcid.org/0000-0002-0901-4615 Xiqing Yue ๑ https://orcid.org/0000-0001-5558-1461 